Original Research Paper

\title{
Omni-Zernike Algorithm for Template Matching in Catadioptric System
}

\author{
${ }^{1}$ Anisse Khald, ${ }^{2}$ Amina Radgui and ${ }^{1}$ Mohammed Rziza \\ ${ }^{1}$ LRIT, Rabat IT Center, Faculty of Sciences, Mohamed V University, B.P.1014 RP, Rabat, Morocco \\ ${ }^{2}$ National Institute of Posts and Telecommunications, INPT, Rabat, Morocco
}

\author{
Article history \\ Received: 06-10-2020 \\ Revised: 29-12-2020 \\ Accepted: 30-12-2020 \\ Corresponding Author: \\ Anisse Khald \\ LRIT, Rabat IT Center, Faculty \\ of Sciences, Mohamed V \\ University, B.P.1014 RP, \\ Rabat, Morocco \\ Email: khald.anisse@gmail.com
}

\begin{abstract}
Image descriptor have been widely applied in many computer visions and image understanding applications including pattern recognition, robotic, video surveillance, camera calibration and image retrieval, etc. Invariants features are robust when apply several transformations of photometry (illumination, blur, noise, JPEG compression) and transformations of geometry (scaling, rotation, translation and viewpoint change). In this study, we present representation and matching region descriptors. Consequently, a set of region used provided by catadioptric system for evaluation of the performance. These regions are normalized by unit circle form with form and size change. In this contribution, the image descriptors of regions used is Moment's Zernike. They are most suitable invariants in omnidirectional context thanks to the polar coordinates used both omnidirectional geometry and Zernike Moments formulation. The aim is realize a robust matching between object's block by using a measure of distance between Zernike's moment descriptors for optimal similarity. Results shown clearly demonstrate the performance of our method and powerful than most important region descriptors (GLOH, SIFT, PCA-SIFT, complex moments and steerable filters) in term of the ROC curve or precision-recall criterion.
\end{abstract}

Keywords: Zernike Moment, Block Matching, Omnidirectional Images

\section{Introduction}

In Computer vision, we present a method for processing, analysing and understanding images system. The aim is giving us much information about environment. The use of Catadioptric camera mounted in mobile robot or installed in monitoring scene is an advantage. In one hand, due to giving us maximum informations about scene and in the other hand the tracking be-come so easy in processing after adapting the suitable algorithm. Object tracking is a challenging task in computer science applications like video surveillance, Radar, mobile robotics, scene matching, so on. This work is focus on processing of omnidirectional images sequence. This is a difficult task because these images have significant distortions in geometry. This is why, we most take them into account during processing.

This work has two main goals. The first one is evaluate an adapted process using polar coordinates over the image plane and unit circle. The second is made a non-rigid object tracking by minimization of the distance between Zernike moments features.

In context of pattern recognition, Invariants Moments is one of popular local image representation and matching that much used in several applications such as video surveillance, image analysis.

The omnidirectional image provided by a catadioptric camera with a Single View Point ((SVP) Teague, 1980; Hu, 1962; Mukundan et al., 2001) is presented by a spherical image (Fig. 1). This model called unified projection model and was defined in fist time in (Teague, 1980).

Following the parabolic mirror shape used in this system, the projection process onto the unit sphere and the catadioptric plan is shown in Fig. 2.

The 3D point $P$ (Equation 1) is projected onto the sphere in.

This point after that will be changed to the new reference frame centred in $P_{m}$ by using the camera parameter $(\epsilon)$. This last ranges between 0 (planar mirror) and 1 (parabolic mirror). 


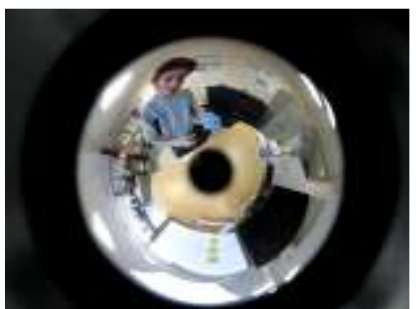

(a)

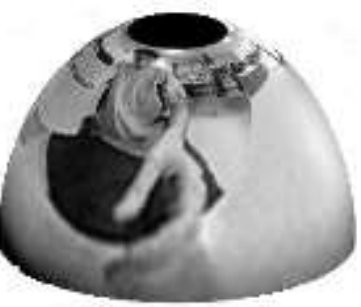

(b)

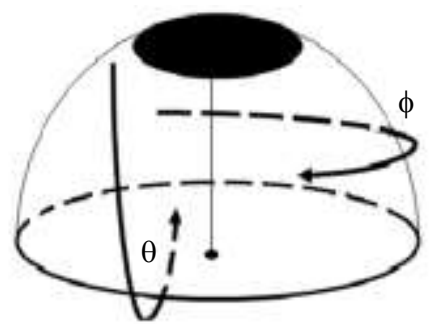

(c)

Fig. 1: Image geometry (a) 2D Omnidirectional Image (b) 3D Spherical Image (c) 3D spherical coordinates

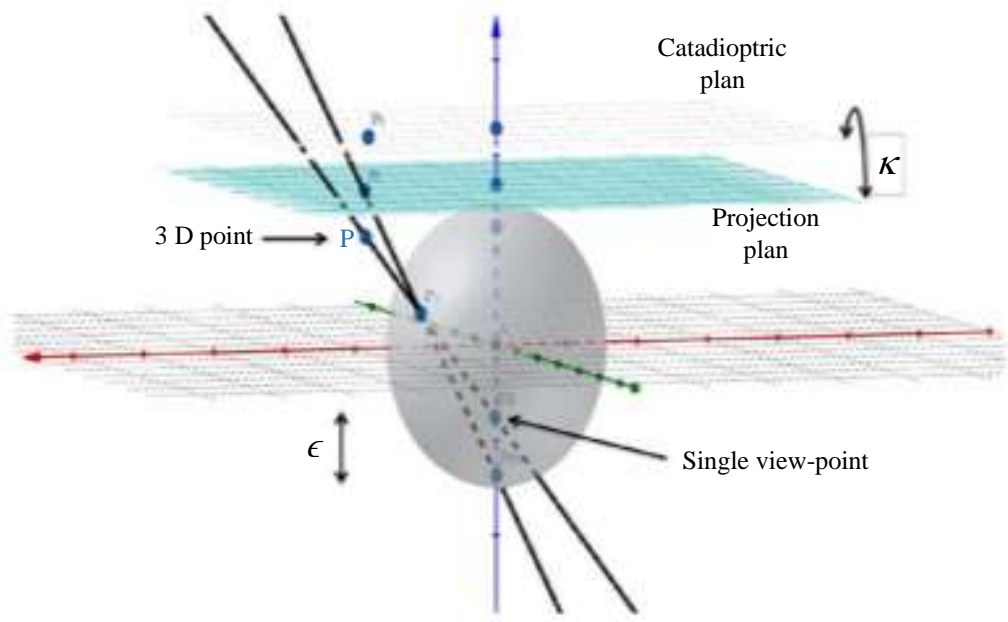

Fig. 2: Unified projection model for central catadioptric cameras of Geyer and Daniilidis (Teague, 1980)

Then, the point $P_{m}:\left(x_{s} ; y_{s} ; z_{s}-\epsilon\right)$ is projected onto the normalized plane using coordinate system with point $m=$ $(u ; v ; 1)$. The point $P_{i}$ is computed by $p_{i}=\kappa * m$. Where $\kappa$ is a matrix tree dimension that contain the intrinsic parameters of camera. The $\kappa$ matrix and the $\epsilon$ parameter are computed by the calibration process. In our work we used a parabolic mirror with $\epsilon=1$ and $\kappa=f\left(\alpha_{u}, \alpha_{v}, u_{0}\right.$, $\left.v_{0}\right)$. In this study, we consider that our Catadioptric camera is already calibrated.

Spherical coordinates of $P_{s} p h$ are shown in Equation (1):

$$
\left\{\begin{array}{l}
X_{s} p h=\cos (\varphi) \sin (\theta) \\
Y_{s} p h=\sin (\varphi) \sin (\theta) \\
Z_{s} p h=\quad \cos (\theta)
\end{array}\right.
$$

The stereographic projection of $P_{s} p h$ from the sphere onto catadioptric plane can be depicted by Cartesian coordinates in Equation (2):

$$
\left\{\begin{array}{l}
u=\frac{X_{s} p h}{1-Z_{s} p h} \\
v=\frac{Y_{s} p h}{1-Z_{s} p h}
\end{array}\right.
$$

Using Equation (1) and (2), we have the image point $P(u, v)$ according to spherical coordinates as Equation (3):

$\left\{\begin{aligned} u & =\cot \frac{\theta}{2} \cos (\varphi) \\ v- & =\cot \frac{\theta}{2} \sin (\varphi)\end{aligned}\right.$ 
where, $\theta$ is the latitude ranges between 0 and $\pi$ and $\varphi$ is the longitude varying between 0 and $2 \pi$. The spherical point according to spherical coordinates system is presented by $(\theta, \varphi)$.

The current paper is writing according to the plan as follows. In part II, we give the mathematical theory of invariant moments for scale and rotation change. In part III introduce the radial Zernike moments in our context and discusses how the proposed invariant sets addresses the classical processing in case of omnidirectional images. Section IV examines the performance of the proposed radial central moments and Zernike central moments. Finally, concludes the study by showing a comparison in the experimental studies between radial moments of Zernike moments and the classical approach.

\section{Related Works}

In the literature, we find examples of moment-based feature descriptors such as geometric, rotational, orthogonal and complex moments. Orthogonal moments defined in terms of a set of orthogonal basis are often preferred due greatly to its ability to represent images with the minimum amount of information redundancy. Teague (1980) proposed Zernike moments based on the basis set of orthogonal Zernike polynomials. It is well known that a discrete image function can be reconstructed by Zernike moments (Liao and Pawlak, 1998). Khotanzad and Liou (1996) used Zernike moment in-variants in recognition and pose estimation of threedimensional objects. Belkasim et al. $(1989 ; 1991)$ did a comparative study on Zernike moment invariants and used them in shape recognition. Vengurlekar et al. (2019) used Zernike moment in object tracking by using comparison between descriptor. Also in (Górniak and Skubalska-Rafajłowicz, 2017) those moment are used in classification. Ghosal and Mehrotra (1993) use Zernike moments in composite-edge detection of threedimensional objects. Zhou et al. (2016), Zernike moment are used also in object tracking by using distance between moments. Particularly, the Zernike moments have been shown to be rotation invariance and noise robust. The low order moments represent the global shape of a pattern and the higher order the detail.

\section{Complex Zernike Moments}

Zernike moment has been introduced based on a continue orthogonal function called Zernike polynomials. The zernike moment applied in digital image can be computed by using (4) (Zhang et al., 2010; Perantonis and Lisboa, 1992). $f(x, y)$ is the image pixel density. PxQ image size:

$$
Z_{P Q}=\frac{n+1}{\pi} \sum_{k=p}^{q} B_{q p k} \sum_{x=1}^{Q} \sum_{y=1}^{P}(x-i y)^{p}\left(x^{2}+y^{2}\right)^{\frac{k-p}{2}} f(x, y)
$$

where:

$$
B_{q p k}=\frac{(-1)^{(q-k)_{2}}\left(\frac{q+k}{2}\right) !}{\left(\frac{q-k}{2}\right) !\left(\frac{k+p}{2}\right) !\left(\frac{k-p}{2}\right) !}
$$

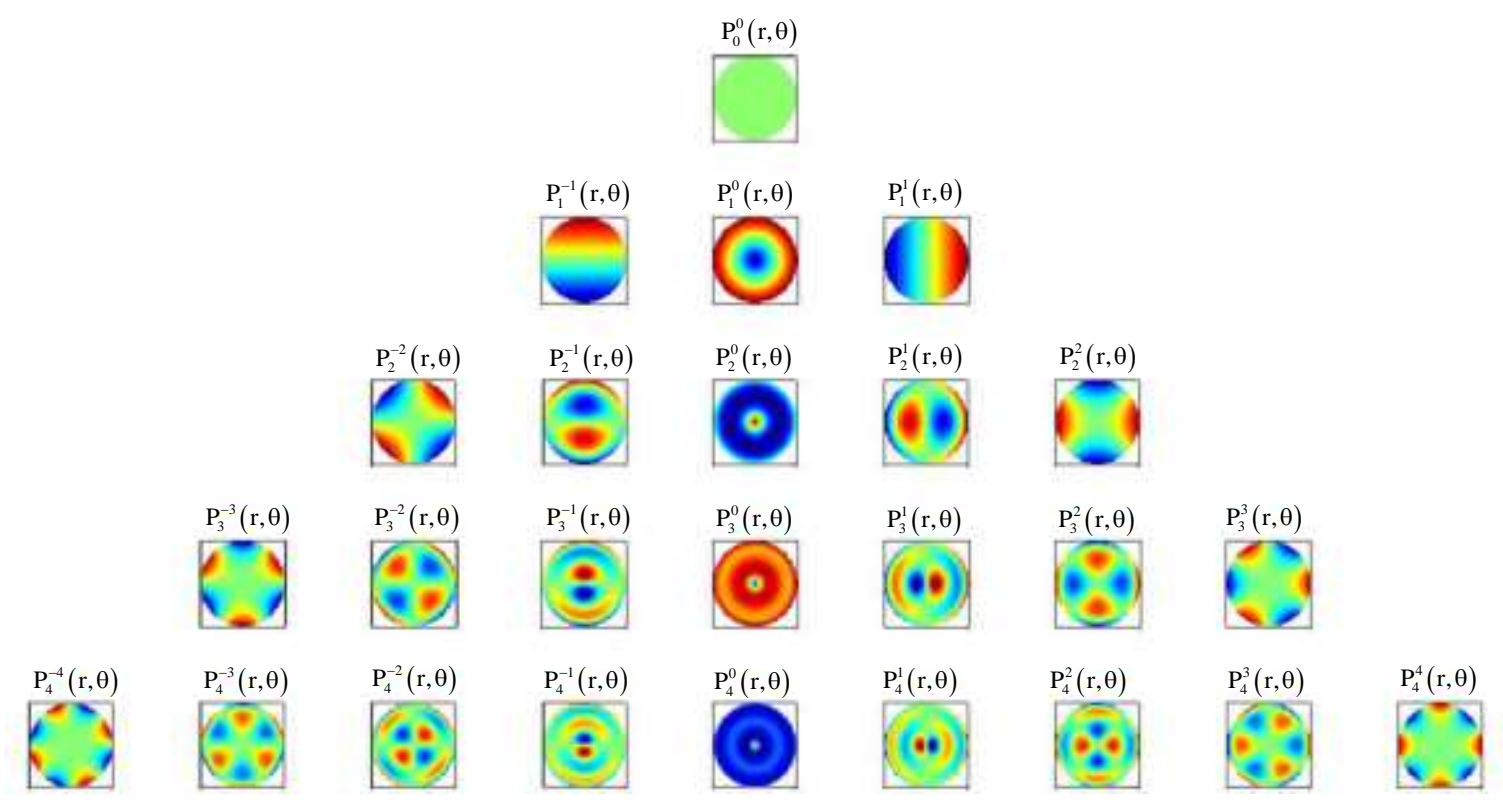

Fig. 3: Plots of $P_{p q}(r, \theta)$ in different $p$ and $q$ 
Equations for Zernike moments for rotation and scaling factors can be computed by using (4) by a substitution from (5). The magnitude $\left|Z_{p q}\right|$ of the Zernike moment can be taken as a rotation invariant feature of the underlying image function (Saad and Rusli, 2004) (Fig.3):

$$
\begin{aligned}
& Z_{20}=\left(\frac{3}{\pi}\right)\left(2\left(\eta_{20}+\eta_{02}\right)-\eta_{00}\right) \\
& \left|Z_{22}\right|^{2}=\left(\frac{3}{\pi}\right)^{2}\left[\left(\eta_{20}-\eta_{02}\right)^{2}+4 \eta_{11}^{2}\right] \\
& \left|Z_{31}\right|^{2}=\left(\frac{12}{\pi}\right)^{2}\left[\left(\eta_{30}-\eta_{12}\right)^{2}+\left(\eta_{21}+\eta_{03}\right)^{2}\right] \\
& \left|Z_{33}\right|^{2}=\left(\frac{4}{\pi}\right)^{2}\left[\left(\eta_{30}-\eta_{12}\right)^{2}+\left(\eta_{21}+\eta_{03}\right)^{2}\right] \\
& \left|Z_{42}\right|^{2}=\left(\frac{5}{\pi}\right)^{2}\left(\left[4\left(\eta_{40}-\eta_{04}\right)^{2}-3\left(\eta_{20}+\eta_{02}\right)^{2}\right]+\left[6 \eta_{11}-8\left(\eta_{31}-\eta_{13}\right)\right]^{2}\right) \\
& \left|Z_{42}\right|^{2}=\left(\frac{5}{\pi}\right)^{2}\left(\eta_{00}-6\left(\eta_{20}+\eta_{02}\right)+6\left(\eta_{40}+\eta_{04}+2 \eta_{22}\right)\right)
\end{aligned}
$$

We have to use $\mathrm{ZM}$ phase and module information both to make the region descriptor. Let the Zernike moments be sorted by $p$ and $q$ in order. The total number of complex ZM moments of the same repetition $m$ is equal to $\frac{N-m}{2}+1$.

Table 1 gives the $42 \mathrm{ZM}$ moments where the maximum order $p$ and maximum repetition $q$ are both equal to 12. The sorted Zernike moments list form a feature vector as follows:

$$
P=\left[Z_{11}\left|e^{i \phi_{11}}, Z_{13}\right| e^{i \phi_{13}}, \ldots, Z_{N M} \mid e^{i \phi_{N M}}\right]
$$

Errors caused in Zernike moment process using Equation (4) due to the using Cartesian coordinates ( $x$, $y$ ) in processing. Which is justified by the fact that images are represented by square pixels. However, this approach does not take into account the radial kind of Zernike polynomials that use omnidirectional image geometry (Fig. 4).

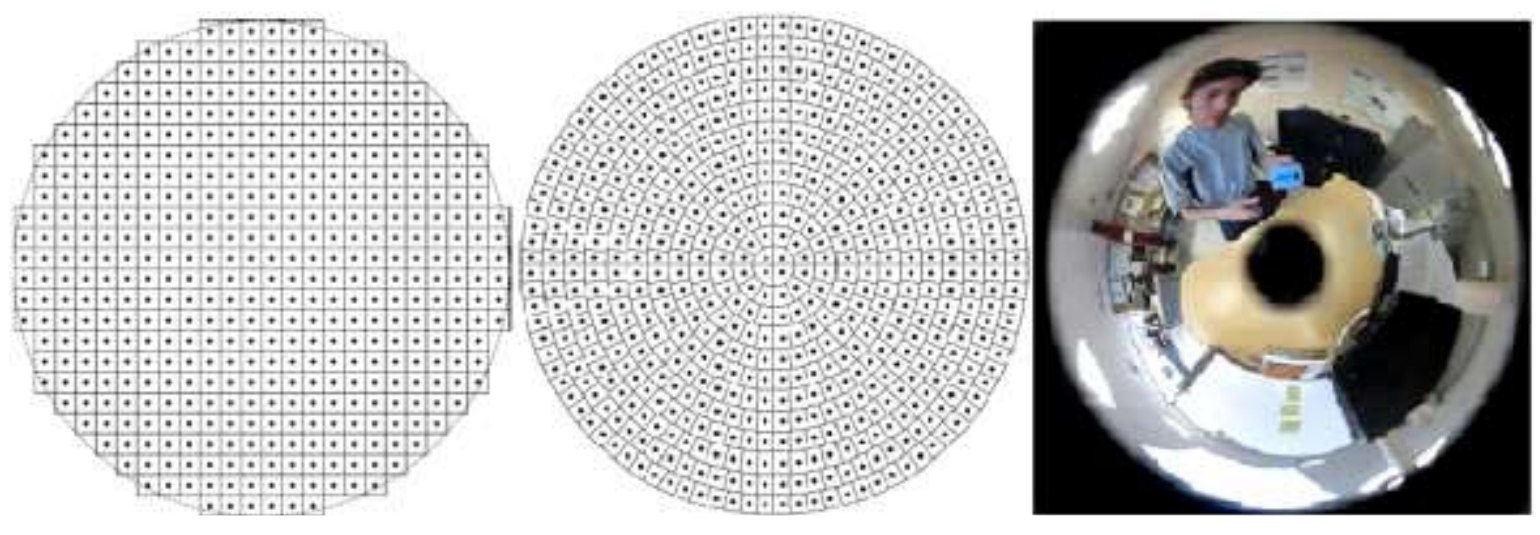

Fig. 4: Polar pixel tiling scheme for ZMs

Table 1: ZMs listed by $p(p, q)=(12,12)$

\begin{tabular}{llllll}
\hline $\mathrm{p}$ & Moments & No. & $\mathrm{p}$ & Moments & No. \\
\hline 1 & $Z_{11}, Z_{31}, Z_{51}, Z_{71}, Z_{91}, Z_{11,1}$ & 6 & 7 & $Z_{77}, Z_{97}, Z_{11,7}$ & 3 \\
2 & $Z_{22}, Z_{42}, Z_{62}, Z_{82}, Z_{10,2}, Z_{12,2}$ & 6 & 8 & $Z_{88}, Z_{10,8}, Z_{12,8}$ & 3 \\
3 & $Z_{33}, Z_{53}, Z_{73}, Z_{93}, Z_{11,3}$ & 5 & 9 & $Z_{99}, Z_{11,9}$ & 2 \\
4 & $Z_{44}, Z_{64}, Z_{84}, Z_{10,4}, Z_{12,4}$ & 5 & 10 & $Z_{10,10}, Z_{12,10}$ & 2 \\
5 & $Z_{55}, Z_{75}, Z_{95}, Z_{11,5}$ & 4 & 11 & $Z_{11,11}$ & 1 \\
6 & $Z_{66}, Z_{86}, Z_{10,6}, Z_{12,6}$ & 4 & 12 & $Z_{12,12}$ & 1 \\
\hline
\end{tabular}

In this part, we present an method for Zernike Moment computing according to polar coordinates $(x, y$, $z=0)$. As follows The radial moments with order number $p$ and repetition $q$ in radial case are defined as:

$$
D_{p q}=\int_{\theta=0}^{2 \pi} \int_{r=0}^{\infty} r^{p} e^{-j q \theta} d r d \theta, j^{2}=-1
$$

- The polar pixels should be as 'square' as possible. The sector boundary lengths must be close enough
- Polar pixels must be organized as regularly as possible to facilitate computing

- The unit circle is uniformly divided along the radial direction in sections

where, $p=0,1,2, \ldots, 1$ and $q$ takes on any positive or negative integer values. The kernel of Zernike moments is a function of orthogonal Zernike polynomials defined over the polar coordinate space 
inside a unit circle such us the omnidirectional image normalized. The two-dimensional Zernike moments of order $p$ with repetition $q$ of an image intensity function $f(r, \theta)$ are defined as (Khotanzad and Liou, 1996):

$$
Z_{p q}=\frac{p+1}{\pi} \int_{\theta=0}^{2 \pi} \int_{r=0}^{\infty} V^{*}(r, \theta) f(r, \theta) r d r d \theta,|r| \leq 1
$$

where, Zernike polynomials of order $p$ with repetition $q$, $P_{p q}(r, \theta)$, are defined as:

$$
P_{p q}(r, \theta)=R_{p q} e^{j q \theta}
$$

and the real valued radial polynomial, $R_{p q}(r)$, is given as follows:

$$
R_{p q}(r)=\sum_{k=0}^{p-\frac{|q|}{2}}(-1)^{k} \frac{(p-k) !}{k !\left(\left(\frac{p+|q|}{2}-k\right) !\left(\frac{p-|q|}{2}-k\right) !\right)}
$$

In omnidirectional context, the image presented by his polar coordinates figure ?? like zernike moment in the unit circle.

\section{Improved Moment in Rotation and Scale Invariant}

In Fig. 5 and 6, we present the invariance in term of rotation ans scale of all Zernike moment module. It means that Zernike moments are the suitable moments in our case due to rotation in omnidirectional images and scale change when the object is moving to the camera or from.

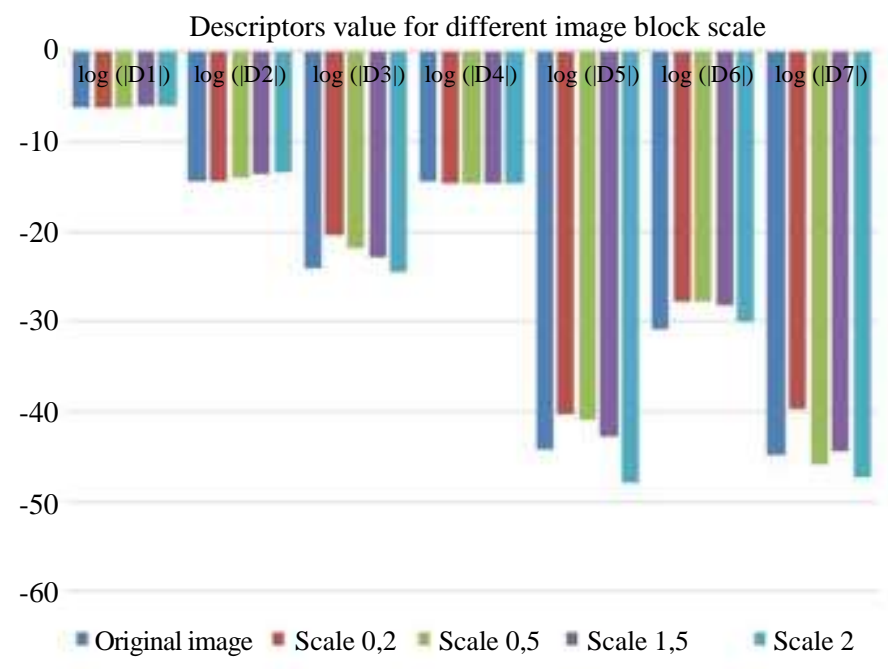

Fig. 5: Scale invariant

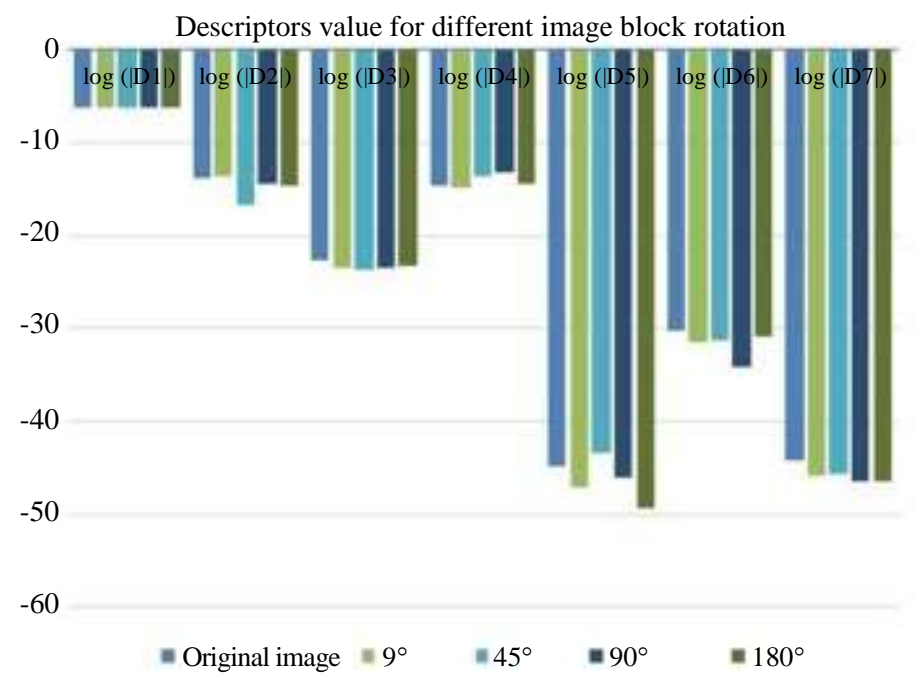

Fig. 6: Rotation invariant 


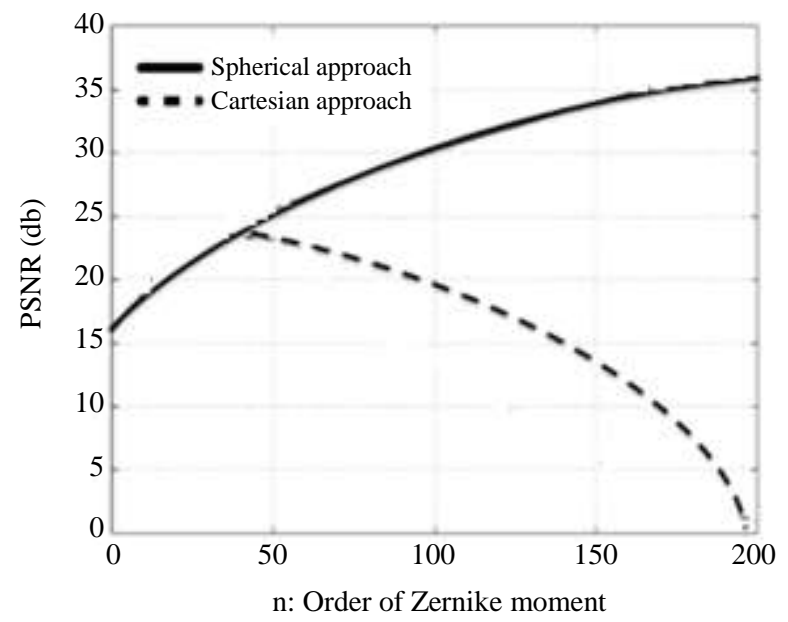

Fig. 7: Image invariance in term of PSNR

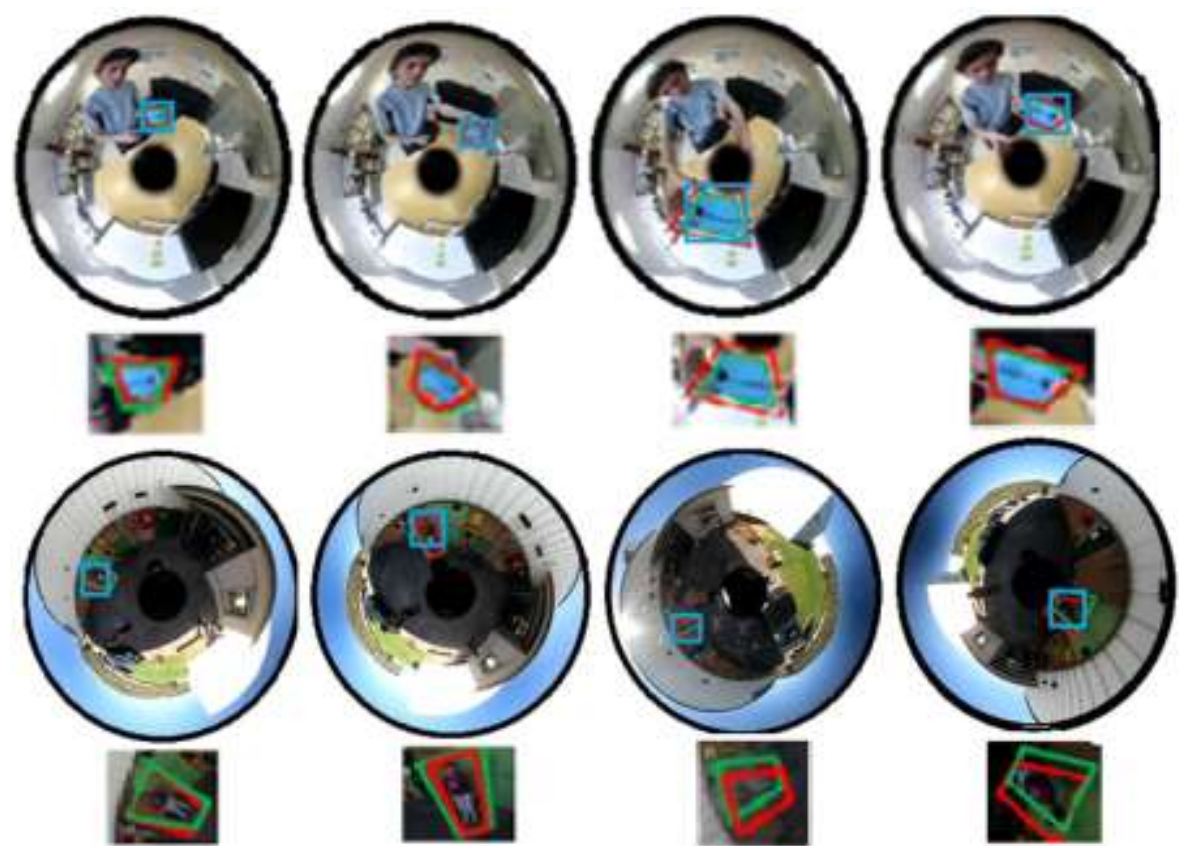

Fig. 8: Tracking results. Tracking results compared with the conventional case depicted in blue. Results with the proposed method are shown in red and the ground truth is in green

Also in term of PSNR, our method is mush better than the Cartesian approach (Fig. 7).

\section{Similarity Measurement}

\section{Classical Approach}

The usual measure to compare two descriptors of Zernike is a simple Euclidean distance between the duels moments:

$$
d^{2}=\sum \sum_{(p, q) \in D}\left(\left|Z_{p q}\right|-\left|Z_{p q}\right|^{\prime}\right)^{2}
$$

We designate this distance as the classical distance. It is based on moment modules (two-image block are considered identical since their modules are the same). Consequently, we have a loss of information without using phase moment to recover the angle of rotation between the images blocks because this information is coded on the moment phase.

The new measure takes account of this issue. This similarity score is more robust than the classical method and recovers a rotation angle between the two images blocks. The angle is optimal when the Euclidean distance between the current block and the next will be minimized: 


$$
d_{I, J}^{2}(\theta)=\sum_{(r, \theta)} \sum\left[\begin{array}{l}
\sum_{(p, q) \in D} \sum Z_{p q}^{I} \cdot V_{p q}(r, \theta) \\
-\sum_{(p, q) \in D} \sum Z_{p q}^{I} e^{i q \theta} \cdot V_{p q}(r, \theta)
\end{array}\right]
$$

\section{Results}

We note that the conventional approach in this case in applying the distance and the moments in Cartesian case. We just applied the classical process directly in omnidirectional image sequence. Results shown in Fig. 8 that out method address this problem of template matching better than the classical because we must take into account the deformed geometry of images provided by catastrophic system.

\section{Conclusion}

In this study, we present a template matching method between blocks modelled by Zernike moments parameters. This process is done by computing a new similarity measure. We optimize this distance between models for finding our object after move. Experimental results show the good performance of our approach. A direction of future work would be an extension for object tracking with real time processing.

\section{Acknowledgment}

Omnidirectional image sequence in this study was provided by Cedric Demonceaux, L2i Laboratory, Bourgogne Franche-Comte University.

\section{Author's Contributions}

Anisse Khald: Participated in all experiments and tests and data-analysis. Contributed to the writing of the manuscript.

Amina Radgui: Conceptualization, supervision and investigation.

Mohamed Rziza: Review, editing and formal analysis.

\section{Ethics}

This article is original and contains unpublished work and results. The corresponding author concerns that all of the other authors have read and approved the manuscript and has no ethical issue involved.

\section{References}

Belkasim, S. O., Shridhar, M., \& Ahmadi, M. (1989, January). Shape redognition using Zernike moment invariants. In Twenty-Third Asilomar Conference on Signals, Systems and Computers, 1989. (Vol. 1, pp. 167-168). IEEE Computer Society.
Belkasim, S. O., Shridhar, M., \& Ahmadi, M. (1991). Pattern recognition with moment invariants: a comparative study and new results. Pattern recognition, 24(12), 1117-1138.

Ghosal, S., \& Mehrotra, R. (1993). Orthogonal moment operators for subpixel edge detection. Pattern recognition, 26(2), 295-306.

Górniak, A., \& Skubalska-Rafajłowicz, E. (2017, June). Object Classification using sequences of Zernike moments. In IFIP International Conference on Computer Information Systems and Industrial Management (pp. 99-109). Springer, Cham.

$\mathrm{Hu}$, M. K. (1962). Visual pattern recognition by moment invariants. IRE transactions on information theory, 8(2), 179-187.

Khotanzad, A., \& Liou, J. H. (1996). Recognition and pose estimation of unoccluded three-dimensional objects from a two-dimensional perspective view by banks of neural networks. IEEE Transactions on Neural networks, 7(4), 897-906.

Liao, S. X., \& Pawlak, M. (1998). On the accuracy of Zernike moments for image analysis. IEEE transactions on pattern analysis and machine intelligence, 20(12), 1358-1364.

Mukundan, R., Ong, S. H., \& Lee, P. A. (2001). Image analysis by Tchebichef moments. IEEE Transactions on image Processing, 10(9), 1357-1364.

Perantonis, S. J., \& Lisboa, P. J. (1992). Pattern Recognition by High-Order Neural Networks and Moment Classifiers.

Saad, P., \& Rusli, N. (2004). Feature extraction of trademark images using geometric invariant moment and zernike moment-a comparison.

Teague, M. R. (1980). Image analysis via the general theory of moments. JOSA, 70(8), 920-930.

Vengurlekar, S. G., Jadhav, D., \& Shinde, S. (2019, July). Object detection and tracking using zernike moment. In 2019 International Conference on Communication and Electronics Systems (ICCES) (pp. 12-17). IEEE.

Zhang, H., Dong, Z., \& Shu, H. (2010, March). Object recognition by a complete set of pseudo-Zernike moment invariants. In 2010 IEEE International Conference on Acoustics, Speech and Signal Processing (pp. 930-933). IEEE.

Zhou, Z., Liu, P., Chen, G., \& Liu, Y. (2016, December). Moving object detection based on zernike moments. In 2016 5th International Conference on Computer Science and Network Technology (ICCSNT) (pp. 696-699). IEEE. 